\title{
Fundamental study of lower limb muscle activity using an angled whole body vibration exercise instrument
}

\author{
Chang Ho $\mathrm{Yu}^{\mathrm{a}}$, Seung Rok Kang ${ }^{\mathrm{b}}$ and Tae Kyu Kwon ${ }^{\mathrm{a}, *}$ \\ ${ }^{a}$ Division of Biomedical Engineering, Chonbuk National University, Deokjin-Dong 1-Ga, Jeonju, \\ Jeonbuk 561-756, South Korea \\ ${ }^{b}$ Department of Healthcare Engineering, Chonbuk National University, Deokjin-Dong 1-Ga, Jeonju, \\ Jeonbuk 561-756, South Korea
}

\begin{abstract}
This research was performed to assess the effects of angled whole body vibration on muscle activity of the lower limbs, by examining adults in their twenties during squat exercises, taking into account two variables of exercise intensity (vibration frequency and gradient). Twenty healthy males in their twenties with previous experience of more than 6 month's weight training and no past medical history were included in this study. The experiment was performed by participating in squat exercises which consisted of 3 sets ( 1 set $=5$ seconds $\times 3$ repetitions of exercise), and the muscle activities of the Rectus Femoris, Vastus Lateralis, Vastus Medialis were measured with variation in the gradients of $0^{\circ}, 10^{\circ}$, and $20^{\circ}$, and vibration frequencies of 20,30 , and $40 \mathrm{~Hz}$. At 30 and $40 \mathrm{~Hz}$, the vastus lateralis showed the highest change in muscle activity, while activity of the vastus medialis also increased significantly. Analysis of muscle activity according to the gradient showed a significant increase of the vastus lateralis at $20^{\circ}$, while the highest muscle activity at $20^{\circ}$ was observed for the vastus medialis. In comparison of the change in lower limb muscle activity according to simultaneous stimulation, at a gradient of $10^{\circ}$, high activity was shown in muscle, while at $20^{\circ}$, high muscle activities were produced at $40 \mathrm{~Hz}$ in the vastus lateralis, $40 \mathrm{~Hz}$ in the rectus femoris, and both 30 and $40 \mathrm{~Hz}$ in the vastus medialis
\end{abstract}

Keywords: Lower limb, muscle activity, exercise instrument for whole body vibration

\section{Introduction}

In the modern age, there have been increased demands on health as a result of the development of medical science, and improvements in the quality of life quality and economic state. Hence, modern men exercise using weight machines and other equipment as a cure for stress and to improve health. Weight training is often recommended for its benefits of consuming more energy, providing less risk of injury than other exercises, and helping to improve the body composition, including for the prevention of osteoporosis [1].

Among the different types of weight training, the squat exercise is a representative lower limb strengthening program, which has a large effect on the strength of muscles such as the vastus lateralis,

\footnotetext{
${ }^{*}$ Corresponding author: Tae Kyu Kwon, Division of Biomedical Engineering, Chonbuk National University, Deokjin-Dong 1-Ga, Jeonju, Jeonbuk 561-756, South Korea. Tel.: +82-63-270-4066; Fax: +82-63-270-2247; E-mail: kwon10@jbnu.ac.kr.
}

0959-2989/14/\$27.50 @ 2014 - IOS Press and the authors. 
vastus medialis, bicephalus femoris, and musculus gastrocnemius, also improving bone density, ligaments, and tendons. It is a more efficient training which causes muscular mobilization, stimulating functional patterns and proprioceptive sense [2,3]. The squat has three subsidiaries. The first is bodyweight, using body burden as resistance, the second is a barbell squat, using barbells as resistance, and the final one is a machine squat, adopting a machine as a training tool [2].

Among different tools for providing physical training, whole body vibration (WBV) has been gaining more attention. WBV was recently adopted, in the late 1990s, in the area of muscle function training. It is now widely used in various areas such as in rehabilitation clinics, sports centers, and for professional sport teams in countries such as the USA, Japan, and especially in Europe [4]. The principle of WBV is thus: it improves the function of the neuromuscular system by producing rapid and strong contraction of muscles through stimulation by artificially controlling the gravitational load [5]. The method of controlling gravitational load to strengthen muscles is not new, as improvement of the effects of exercise by adding additional weight to the body or instruments already existed. However, such training can cause injury due to excessive burdens and stimulation to the body, and may also cause secondary effects of over-training due to the overly increased burden. As a solution, there have been many efforts to develop new exercise programs or devices like WBV, which are easy and safe, as well as able to provide different levels of exercise intensity in a controlled way.

One of the related studies was on the effects of WBV on the leg-press performance ability of athletes on a national volley ball team, performed by Dakota, revealing that the leg stimulated by WBV had significantly higher performance than the unstimulated one [6]. Torvinen also examined the effects of vibrational stimulation in his research on vibrational stimulation, postural balance and muscle activity. That study demonstrated enhancement of the soleus muscle, musculus gastrocnemius, and vastus lateralis activity, while also improving postural balance of the body [7]. However, Ruiter reported conflicting results after an 11-week study on subjects in their twenties, indicating that the use of WBV did not affect the improvement of maximum muscle strength or maximum jumping ability [8]. In a similar study on Muscle activity change around the knee according to the gradient and posture changes during squatting of one leg performed on a downward slope, Yu et al. reported the increased muscle activity of the vastus lateralis and vastus medialis [9]. Baek reported the effects of increasing lower limb muscle activity, allowing efficient exercise when heel raising movements were combined with WBV [10].

Studies on exercise with vibration are limited to short or long time training in the squat posture, or on the different effects according to age, while studies on the exercise intensity in accordance with vibration are rare. Hence, this study concentrated specifically on the different effects of two variables of exercise intensity (vibration frequency and gradient) on muscle activity during squat exercises, to provide a more efficient exercise program.

Table 1

Subject information

\begin{tabular}{|l|l|l|l|}
\hline Subjects & $\begin{array}{l}\text { Age } \\
\text { (year) }\end{array}$ & $\begin{array}{l}\text { Height } \\
(\mathbf{c m})\end{array}$ & $\begin{array}{l}\text { Weight } \\
(\mathbf{k g})\end{array}$ \\
\hline $\mathrm{N}=20$ & $20 \pm 0.6$ & $179.7 \pm 1.4$ & $69.0 \pm 6.8$ \\
\hline
\end{tabular}




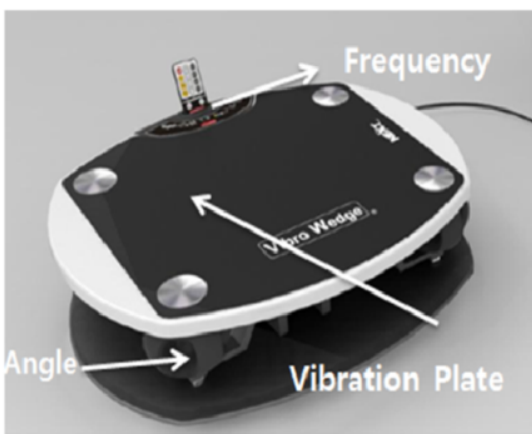

(a)

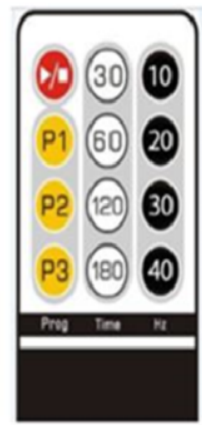

(b)

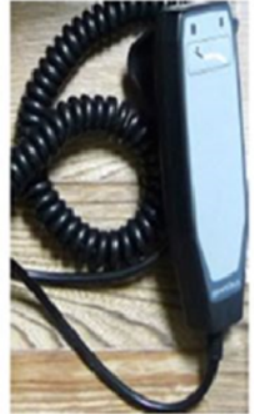

(c)

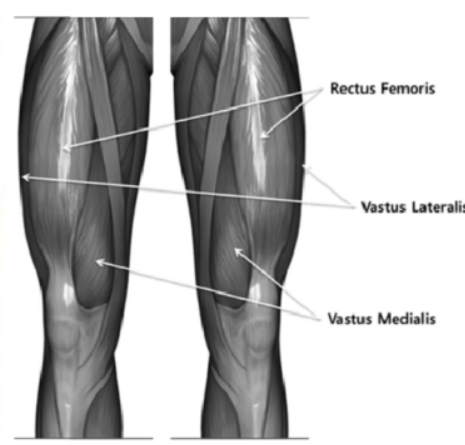

(d)

Fig. 1. (a) Vibro-Wedge (NEXT, Seoul, Korea); (b) Remote control for vibration intensity; (c) Remote control for gradient; and (d) Muscles of the lower limbs measured for verification of muscular activity.

\section{Experimental methods}

\subsection{Subjects}

Table 1 shows the physical information of the subjects. The subjects of this research were all in their twenties and physically healthy, had 6 months of experience in weight training, and had no medical history. They were all informed about the purpose, details, and procedure of the experiment, and voluntarily agreed to participate in the performance of the exercise program for the whole experiment. They were carefully selected to minimize the deviation due to height and weight, to possible reduce errors which might occur.

\subsection{System configuration}

In this study, vibration was performed using a Vibro-Wedge (NEXT, Seoul, Korea), as shown in Figure 1. It can provide different exercise burdens by vertical movements at a frequency of $0-40 \mathrm{~Hz}$, with an amplitude (intensity) of $0.8-1.5 \mathrm{~mm}$. Besides this, it is designed for easy control of gradient (0$25^{\circ}$ ) as well as vibration intensity with the remote control.

\subsection{Experimental procedures}

Figure 2(a) represents the experimental procedure to examine the effects of WBV conducted with variation of two complex exercise intensity variables (vibration frequency and gradient) on muscle activity during squat exercise. All subjects performed squats while standing on an angled vibration plate with bare feet, as shown in Figure 2(b). Specified instructions were given for the squats, to stretch back, look forward, keep knees close to each other, and bend the knees for 5 seconds before standing. While 3 sets ( 1 set being comprised of 3 repetitions) were being performed, each muscle activity was measured under the conditions of gradients at $0^{\circ}, 10^{\circ}$, and $20^{\circ}$, and vibration frequencies of $0 \mathrm{~Hz}, 20 \mathrm{~Hz}, 30 \mathrm{~Hz}$, and $40 \mathrm{~Hz}$. For measurement of muscle activity, the electromyogram (EMG) value was measured for the Rectus Femoris (RF), Vastus Lateralis (VL) and Vastus Medialis (VM) using the Bagnoli-8ch Desktop EMG System (Delsys Inc., NY, USA), as shown in Figure 1(d). The sampling rate was set to $1000 \mathrm{~Hz}$, with a frequency bandwidth of 6-400 Hz, which is the measurement fre 


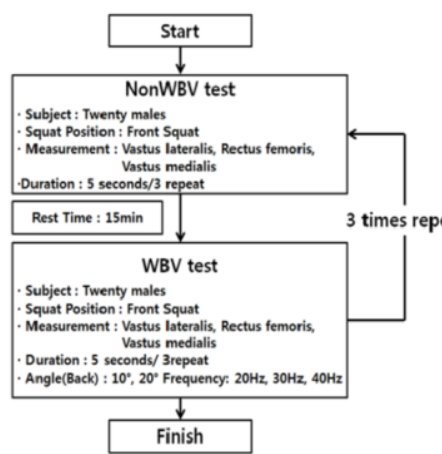

(a)

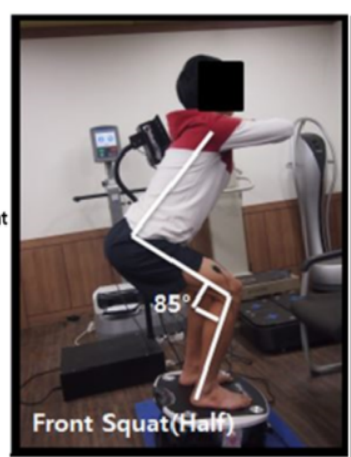

Front Squat(Falf)

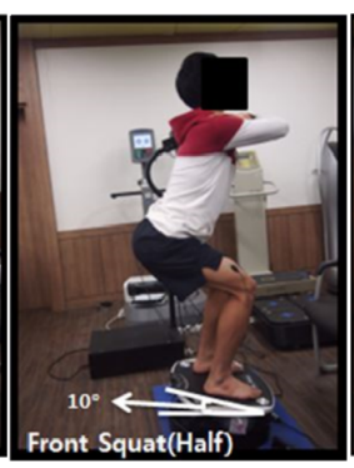

(b)

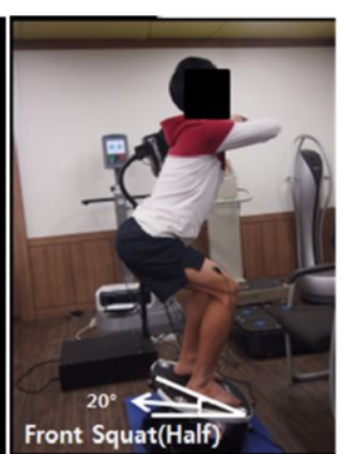

Front Squat(Half)

Fig. 2. (a) Diagram of the experimental procedure and (b) Whole body vibration instrument using various vibration frequencies and gradients during the front squat exercise.

quency bandwidth filter of the Bagnoli EMG System. RMS (Root Mean Square) analysis was applied to analyze the EMG data.

\subsection{Data processing and analysis}

To verify changes in the muscle activity according to vibration intensity and gradient, SPSS 20.0 for Windows (SPSS Inc., Chicago, USA) was used to calculate statistics. The normality test was done through the Paried-Samples t-test to analyze the significance of differences among the averages of the variables. The significance level was set to ${ }^{*} \mathrm{p}<0.05$.

\section{Results}

\subsection{Changes in muscle activity according to vibration intensity}

Figure 3 shows the changes in muscle activity according to vibration intensity. On the $\mathrm{X}$ axis, the vibration intensities of $0 \mathrm{~Hz}, 20 \mathrm{~Hz}, 30 \mathrm{~Hz}$, and $40 \mathrm{~Hz}$ are plotted, while the RMS values of the EMG data are shown on the $\mathrm{Y}$ axis. Figure 3(a) presents the activity of the vastus lateralis, according to the presence or absence of vibration, and the vibrational intensity during the squat movements. When vibration stimulation was given, a significant increase of muscle activity occurred in the vastus lateralis when $40 \mathrm{~Hz}$ was used $(\mathrm{p}<0.05)$. The activity of the vastus medialis was presented in Figure $3(\mathrm{~b})$. Significant increase in the muscle activity was observed for vibration at both 30 and $40 \mathrm{~Hz}(\mathrm{p}<0.05)$.

\subsection{Muscle activity change according to gradient}

Figure 4 presents the change in muscle activity according to the gradient during the squat exercise. The $\mathrm{X}$ axis shows the gradients of $0^{\circ}, 10^{\circ}, 20$, while the $\mathrm{Y}$ axis shows the RMS values of the EMG data. Figure 4(a) presents activity of the vastus lateralis muscle according to the gradient. The result showed a significant increase of muscle activity at a gradient of $20^{\circ}(\mathrm{p}<0.05)$. A significant increase of the muscle activity of the vastus lateralis was also observed at a gradient of $20^{\circ}(\mathrm{p}<0.05)$ (Figure $4(\mathrm{~b})$ ). 


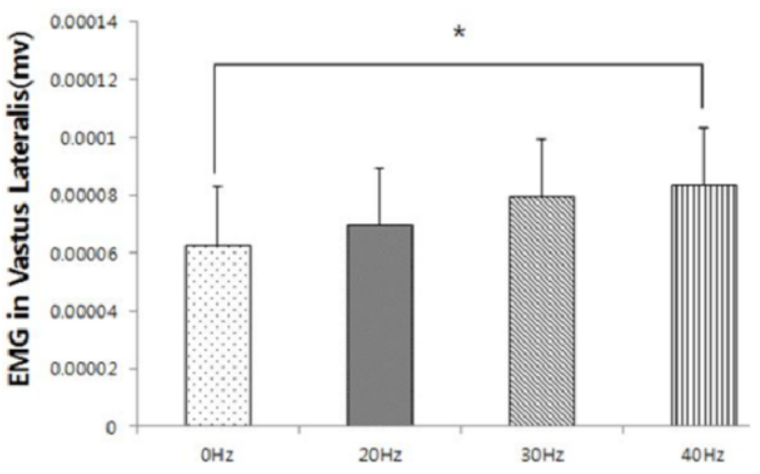

(a)

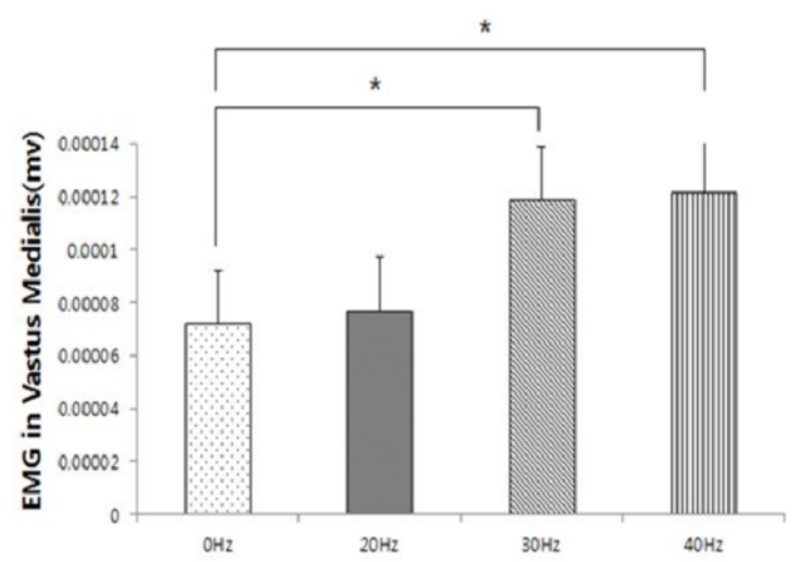

(b)

Fig. 3. (a) Changes in vastus lateralis muscle activity during the front squat exercise according to vibration intensity $\left({ }^{*} \mathrm{p}<0.05\right)$ and $(\mathrm{b})$ Changes in vastus medialis muscle activity during the front squat exercise according to vibration intensity $(* \mathrm{p}<0.05)$.

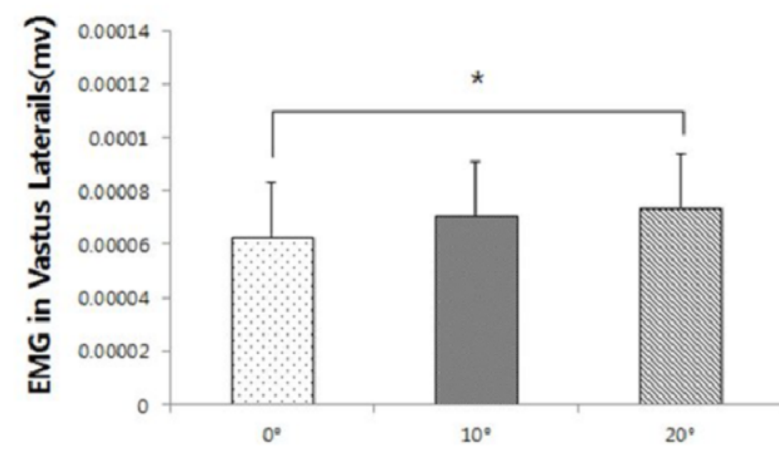

(a)

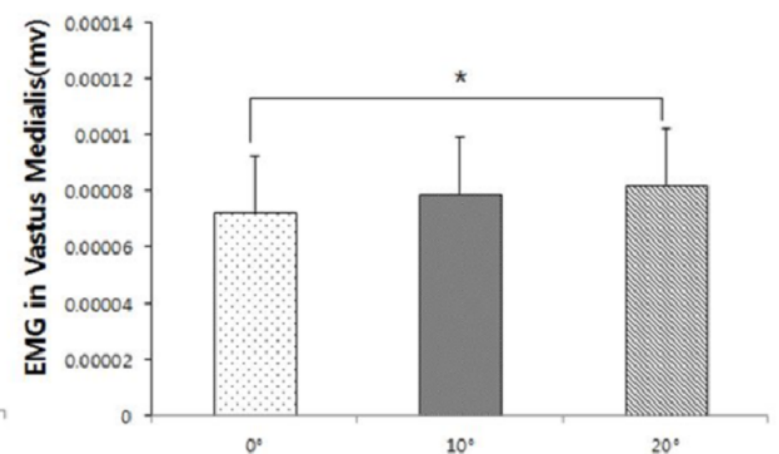

(b)

Fig. 4. (a) Changes in vastus lateralis muscle activity during the front squat exercise according to gradient $\left({ }^{*} \mathrm{p}<0.05\right)$ and $(\mathrm{b})$ Changes in vastus medialis muscle activity during the front squat exercise according to gradient $\left({ }^{*} \mathrm{p}<0.05\right)$.

\subsection{Muscle activity according to simultaneous stimulation (vibration intensity with gradient)}

Figures 5(a), 5(b) and 6(a) show the changes in muscle activity according to the vibration intensity with a gradient of $10^{\circ}$ during squat exercises. The $\mathrm{X}$ axis shows a vibration intensity of $0 \mathrm{~Hz}$ when gradient the gradient was $0^{\circ}$, while the intensities increased from 0 to $40 \mathrm{~Hz}$ along the $\mathrm{X}$ axis for the gradient of $10^{\circ}$. The RMS values of the EMG data are shown on the Y axis. The activity of the vastus lateralis can be seen in Figure 5(a). Muscle activity increased from 15\% to 29.5\% as the vibration frequency increased, when the gradient was fixed to $10^{\circ}$. A significant increase was recorded at $10^{\circ}$ for vibration at $40 \mathrm{~Hz}$ in comparison the other conditions, except for $20 \mathrm{~Hz}(\mathrm{p}<0.05)$. Figure 5(b) shows the activity of the rectus femoris muscle. Muscle activity increased from $0.5 \%$ to $22.7 \%$ as vibration frequency increased, when the gradient was fixed to $10^{\circ}$. At $10^{\circ}$ and a vibration frequency of $40 \mathrm{~Hz}$, a significant increase was detected compared to 0 and $30 \mathrm{~Hz}$ at that gradient $(\mathrm{p}<0.05)$. The muscle activity of the vastus medialis is shown in Figure 6(a), representing an increased from $11 \%$ to $37.4 \%$ as the 
vibration frequency increased with a fixed gradient of $10^{\circ}$. Significant increases were recorded at 30 , and $40 \mathrm{~Hz}(\mathrm{p}<0.05)$.

Figures 6(b), 7(a) and 7(b) show the changes in muscle activity according to vibration intensity when the gradient was fixed to $20^{\circ}$. The $\mathrm{X}$ axis shows a vibration intensity $0 \mathrm{~Hz}$ when the gradient was set to $0^{\circ}$, and vibration intensities increasing from 0 to $40 \mathrm{~Hz}$ when the gradient was set to $20^{\circ}$. The RMS values from the EMG data are shown on the Y axis. Figure 6(b) shows the muscle activity of the vastus lateralis. Muscle activity increased from $11.8 \%$ to $28.7 \%$ as the vibration frequency increased. A significant increase was recorded at $40 \mathrm{~Hz}(\mathrm{p}<0.05)$. The muscle activity of the rectus femoris is shown in Figure $7(\mathrm{a})$. Muscle activity increased from $1.9 \%$ to $22.1 \%$ as the vibration frequency increased, with a significant increase observed at $40 \mathrm{~Hz}(\mathrm{p}<0.05)$. Similarly, muscle activity of the vastus medialis increased from $8.7 \%$ to $37.8 \%$ as vibration frequency increased, also showing a significant increase at $40 \mathrm{~Hz}(\mathrm{p}<0.05)$ (Figure 7(b)).

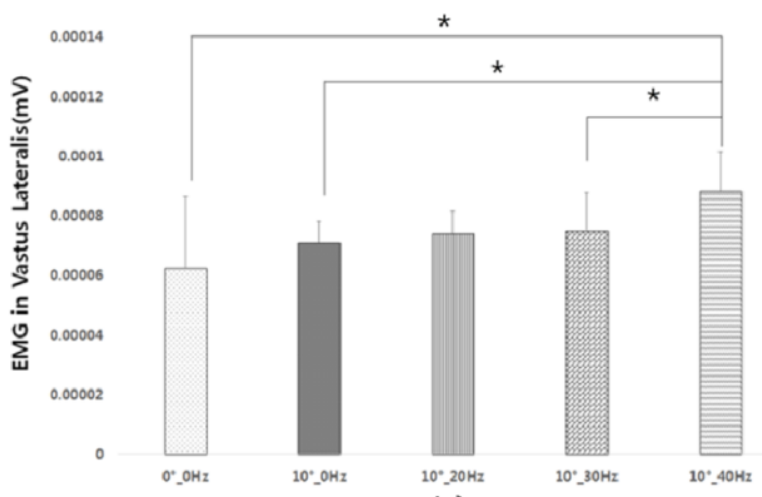

(a)

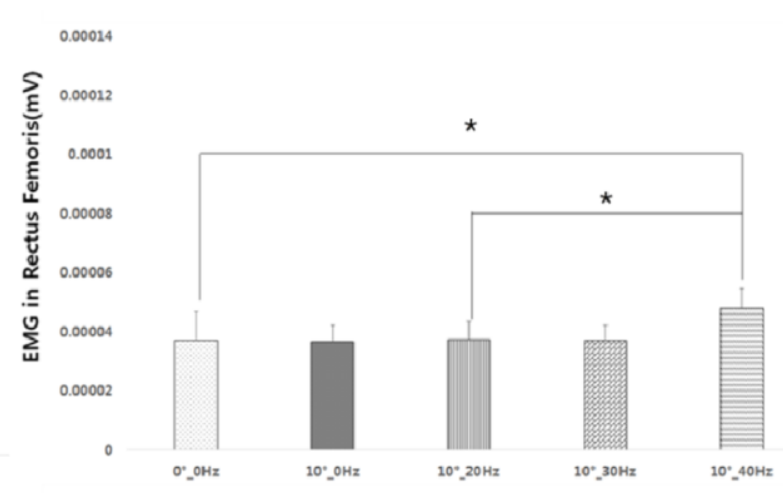

(b)

Fig. 5. (a) Changes in vastus lateralis muscle activity during the front squat exercise according to vibration intensity with a gradient of $10^{\circ}\left({ }^{*} \mathrm{p}<0.05\right)$. (b) Changes in rectus femoris muscle activity during the front squat exercise according to vibration intensity with a gradient of $10^{\circ}\left({ }^{*} \mathrm{p}<0.05\right)$.

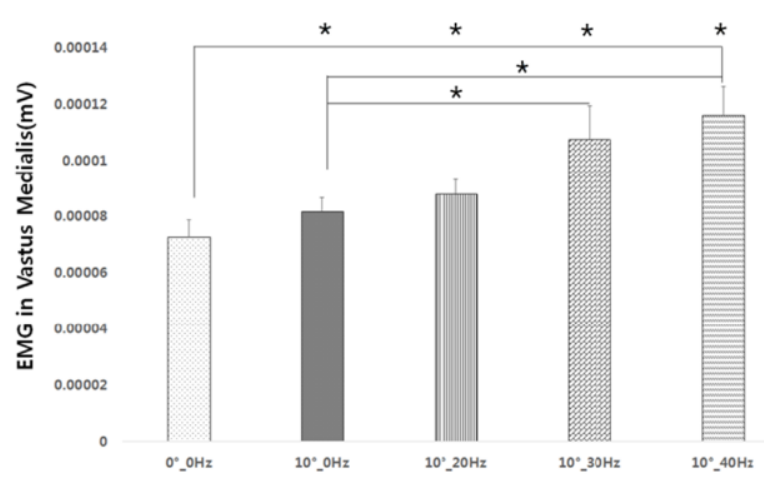

(a)

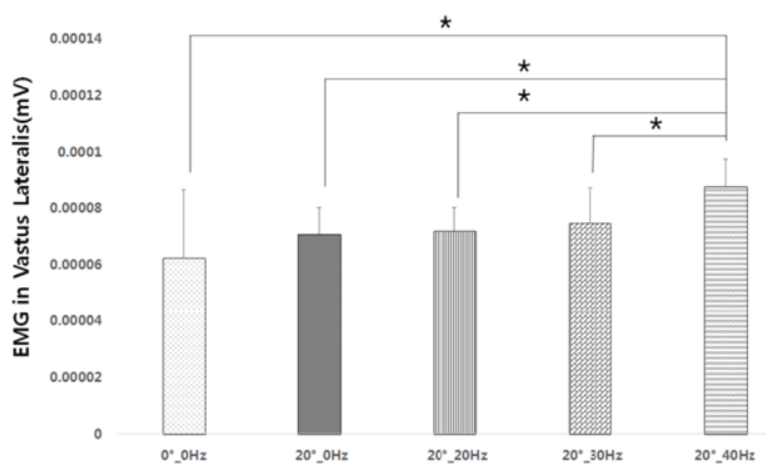

(b)

Fig. 6. (a) Changes in vastus medialis muscle activity during the front squat exercise according to vibration intensity with a gradient of $10^{\circ}\left({ }^{*} \mathrm{p}<0.05\right)$. (b) Changes in rectus vastus lateralis muscle activity during the front squat exercise according to vibration intensity with a gradient of $20^{\circ}\left({ }^{*} \mathrm{p}<0.05\right)$.) 


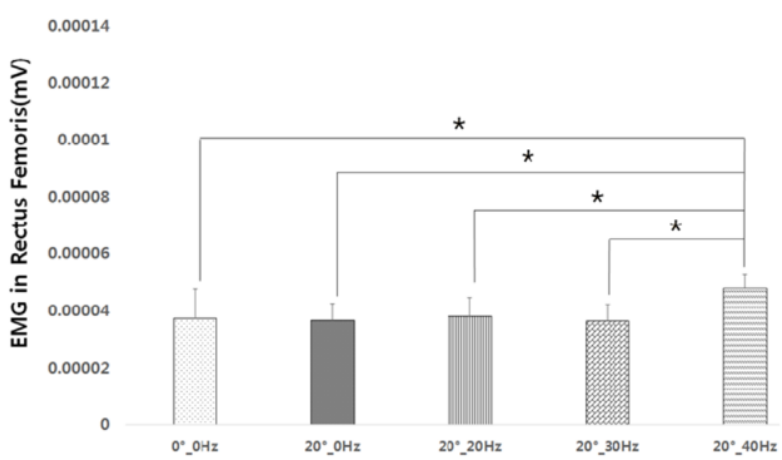

(a)

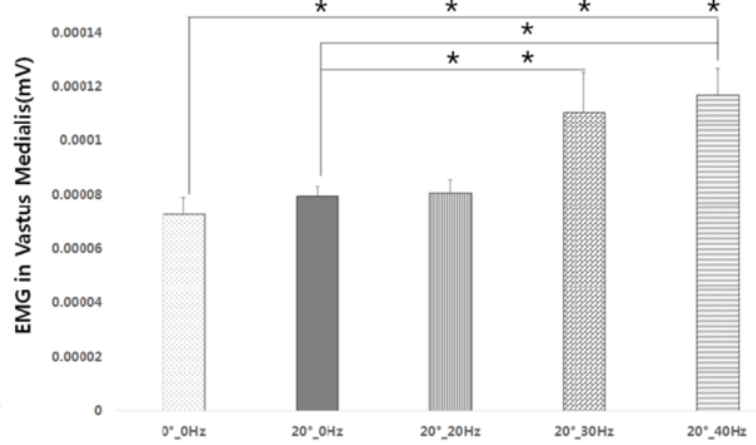

(b)

Fig. 7. (a) Changes in rectus femoris muscle activity during the front squat exercise according to vibration intensity at a gradient of $20^{\circ}\left({ }^{*} \mathrm{p}<0.05\right)$. (b) Changes in rectus vastus medialis muscle activity during the front squat exercise according to vibration intensity at a gradient of $20^{\circ}\left({ }^{*} \mathrm{p}<0.05\right)$.

\section{Discussion}

Analysis of the change in muscle activity according to vibration intensity showed the highest muscle activity changes at $40 \mathrm{~Hz}$ for the vastus lateralis, with significant increases in the vastus medialus at 30 and $40 \mathrm{~Hz}$. However, no significant changes in the rectus femoris were observed, although muscle activity did show an increase. This corresponded with the results of a study by Lee, showing that the vastus medialis and vastus lateralis had an increase in muscle activity as the burden increased during the squat exercise [3]. These results were also similar to those reported by Cadinale, that the activity of the vastus lateralis muscle increased during squat exercise on a WBV instrument [11], while Roelants et al. reported a higher increase in muscle activity in the vastus medialis than those in the rectus femoris and vastus lateralis [12]. It may be conjectured that the bending movement in the squat exercise requires activation of the vastus medialis as well as movement of muscles near the knee, whereas ordinary walking activates the vastus lateralis while deactivating the vastus medialis.

The major factors of WBV which produced positive effects were vibration frequency and amplitude. The experimental results showed the best effects on the vastus lateralis at $40 \mathrm{~Hz}$, and at 30 and $40 \mathrm{~Hz}$ on the vastus medialis; however, this did not correspond to the results of a study by Cardinale that a high vibration frequency of $40 \mathrm{~Hz}$ can cause decline of body function, while a frequency of $20 \mathrm{~Hz}$ can improve muscle function and responsiveness [11]. However, the results were in agreement with those of Rittweger, who advised using a frequency between 20 and $50 \mathrm{~Hz}$, since the use of frequencies below $20 \mathrm{~Hz}$ induce excessive relaxation of muscles, while those over $50 \mathrm{~Hz}$ induce muscle pain and are not recommendable. Similarly, Prisby \& Belli (2008) concluded that the appropriate frequency for WBV to enhance muscle strength and power was over $25 \mathrm{~Hz}[13,14]$. The differences among the results may have occurred from differences in the method for providing vibration or the frequency bandwidth of the instruments, as well as variation in user posture during exercise, given that the vibrational intensity is mostly influenced by factors such as frequency, amplitude, and vibration time.

In comparison of the changes in muscle activity according to the gradient, significant increases were observed in activity of the vastus lateralis, with the highest increase in the vastus medialis when the gradient was set to $20^{\circ}$. Similar to results of vibration frequency, muscle activity also increased in the rectus femoris, but the difference was not statistically significant. The study of Chae et al. showed that 
during squat exercises at $0^{\circ}, 10^{\circ}$, and $20^{\circ}$ angles of tilting the heels, muscle activities in the rectus femoris, vastus lateralis, vasuts medialis increased considerably higher than those of other lower limbs [15]. This result can be interpreted that squat exercise on downward slopes help to erect truncus by balancing the bodily barycenter. In addition, it makes higher muscle activity on the quadriceps femoris muscle by minimizing the influence of the hip muscles, as well as the tibial muscle by using plantar flexion of the ankle joints simultaneously.

Comparison of lower limb muscle activities during simultaneous stimulation with a gradient of $10^{\circ}$ showed that muscle activity had the biggest difference at $40 \mathrm{~Hz}$. Unlike the independent stimulations, simultaneous stimulations significantly increased the activity of the rectus femoris at the $40 \mathrm{~Hz}$ frequency. The vastus medialis showed significant increase in most of the measurements when simultaneous stimulation was provided.

When the gradient was fixed at $20^{\circ}$, high muscle activity was present at $40 \mathrm{~Hz}$ for the vastus lateralis, $40 \mathrm{~Hz}$ for the rectus femoris, and both 30 and $40 \mathrm{~Hz}$ for the vastus medialis. A possible explanation for this would be increase of the positive effect on muscle activity by stimulation of the nerve roots when the WBV was used in independent stimulation, to provide a whole effect. In addition, a downward slope provides another independent stimulation to help the truncus to be erected, while minimizing influence of the hip muscles. All of the factors listed were considered to enable higher muscle activity. Overall, the complex stimulations were more efficient for inducing higher muscle activity in the lower limbs.

\section{Conclusion}

This study was performed to measure and assess the effects of angled whole body vibration on muscle activity in the lower limbs during squat exercise, while taking into account two variables of exercise intensity (vibration frequency and gradient). The results demonstrated the positive effects of angled whole body vibration on muscle activity in the lower limbs. The provision of complex (vibration frequency with gradient) stimulation was found to have more influence, increasing muscle activity of the rectus femoris, vastus lateralis, and vastus medialis at a higher rate than the independent stimulations.

This suggests the possibility that angled whole body vibration may be helpful for training athletes, as well as ordinary people, as it is effective for strengthening muscles. I hope these data will be applied to the development of various angled whole body vibration programs, as well as to the fields of sports and physical education.

\section{Acknowledgment}

This research was financially supported by the Ministry of Trade, Industry and Energy (MOTIE) and Korea Institute for Advancement of Technology (KIAT) through the Promoting Regional Specialized Industry (R0002430).

\section{References}

[1] H.K. Jeong, Electromyographic analyses of the effects of three different plates under heel during squat, Master Degree Dissertation, Kyungpook National University, 2006. 
[2] R.F. Escamilla, Knee biomechanics of the dynamic squat exercise, Medicine \& Science in Sports \& Exercise 33 (2001), $127-141$.

[3] S.D. Lee, J.H. Lee, E.J. Park, K.K. Lee, J.H. Sohn, J.J. Ryue, Y.J. Yu, Y.W. Kim and S.B. Kim, Kinematic, kinetic and EMG pattern during squat exercise in Smith machine with different loads, Korean Journal of Sport Science 2 (2011), 1884-1893.

[4] Y.T. Rhim, The study on whole body vibration as a new exercise training prescription method, Journal of Coaching Development 7 (2005), 105-116.

[5] M. Cardinale and C. Bosco, The use of vibration as an exercise intervention, Exercise and Sport Sciences Reviews 31 (2003), 3-7.

[6] G.F. Dakota, The influence of whole body vibration on jumping performance, Biology of Sport 15 (1998), 157.

[7] S. Torvinen, P. Kannus, H. Sievänen, T.A.H Järvinen, M. Pasanen, S. Kontulainen, T.L.N. Järvinen, M. Järvinen, P. Oja and L. Vuori, Effect of a vibration exposure on muscular performance and body balance: Randomized cross-over study, Clinical Physiology and Functional Imaging 22 (2002), 145-152

[8] C.J. De Ruiter, S.M. Van Raak, J.V. Schilperoort, A.P. Hollander and A. De Haan, The effects of 11 weeks whole body vibration training on jump height, contractile properties and activation of human knee extensors, European Journal of Applied Physiology 90 (2003), 595-600.

[9] W.G. Yoo, C.H. Yi and H.J. Lee, Effects of a combined posture of the lower extremity on activity of the vastus medialis oblique muscle and vastus lateralis muscle during static squat, Physical Therapy Korea 11 (2004), 1-9.

[10] S.W. Baek, Comparative analysis of lower extremity muscle activities for heel raise motion of vibration exercise, Journal of Sport and Leisure Studies 30 (2007), 519-525.

[11] M. Cardinale, R.L. Soiza, J.B. Leiper, A. Gibson and W.R. Primrose, Hormonal responses to a single session of wholebody vibration exercise in older individuals, British Journal of Sports Medicine 44 (2010), 284-288.

[12] M. Roelants, M.P. Sabine, S. Verschueren, C. Delecluse, O. Levin and V. Stijnen, Whole-body-vibration-induce increase in leg muscle activity during different squat exercises, Journal of Strength and Conditioning Research 20 (2006), $124-129$.

[13] J. Rittweger, G. Beller and D. Felsenberg, Acute physiological effects of exhaustive whole-body vibration exercise in man, Clinical Physiology 20 (2000), 134-142.

[14] R.D. Prisby, M.H. Lafage-Proust, L. Malaval, A. Belli and L. Vico, Effects of whole body vibration on the skeleton and other organ systems in man and animal models: what we know and what we need to know, Ageing Research Reviews 7 (2008), 319-329.

[15] W.S. Chae, H.K. Jeong and J.I. Jang, Effect of different heel plates on muscle activities during the squat, Korean Journal of Sport Biomechanics 17 (2007), 113-121. 